\title{
Nomenclatures industrielles en Grèce. Variations du champ du secteur secondaire et limites de comparabilité
}

Industrial nomenclatures in Greece. Variations of the field of the secondary sector and limits of comparability

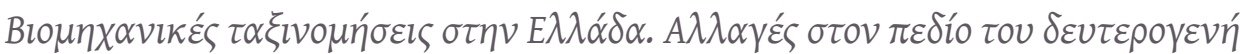

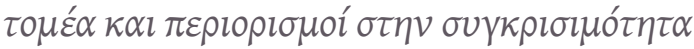

\section{Vincent Gouzi}

\section{OpenEdition}

Journals

Édition électronique

URL : https://journals.openedition.org/ceb/10254

DOI : 10.4000/ceb.10254

ISSN : 2261-4184

\section{Éditeur}

INALCO

Édition imprimée

ISBN : 978-2-85831-294-8

ISSN : 0290-7402

\section{Référence électronique}

Vincent Gouzi, « Nomenclatures industrielles en Grèce. Variations du champ du secteur secondaire et limites de comparabilité », Cahiers balkaniques [En ligne], 45 | 2018, mis en ligne le 14 décembre 2018, consulté le 07 juillet 2021. URL : http://journals.openedition.org/ceb/10254 ; DOI : https://doi.org/ $10.4000 /$ ceb. 10254

Ce document a été généré automatiquement le 7 juillet 2021

\section{cc) (7) 8}

Cahiers balkaniques est mis à disposition selon les termes de la Licence Creative Commons Attribution - Pas d'Utilisation Commerciale 4.0 International. 


\title{
Nomenclatures industrielles en Grèce. Variations du champ du secteur secondaire et limites de comparabilité
}

\author{
Industrial nomenclatures in Greece. Variations of the field of the secondary \\ sector and limits of comparability

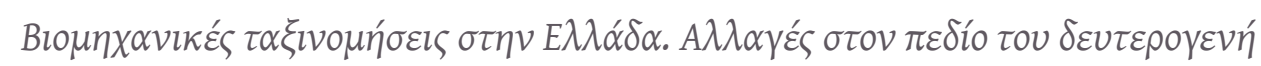

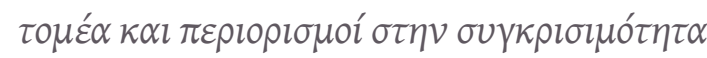

\section{Vincent Gouzi}

1 Nomenclature, classification, taxinomie (en grec), échelle technologique (technologiki klimax)... quel que soit le mot, il désigne ce travail premier du chercheur pour mettre un peu d'ordre dans les produits des activités humaines, dans ce «bazar » qu'évoque Adolphe Quételet ${ }^{1}$. Il le fait pour les outils de la préhistoire (André Leroi-Gourhan), pour les pierres à meules (Margarita Vrettou Souli), pour les outils de la culture du blé en Méditerranée (Pascal Rivet et Serge Luquet) et pour bien d'autres. Une nomenclature est l'inventaire détaillé d'activités dans des temps et des espaces donnés, c'est aussi le moyen de les rendre intelligibles et comparables entre elles.

Pour caractériser la Révolution industrielle, on a construit des nomenclatures. Aussi, pour traiter de l'industrie en Méditerranée, ai-je interrogé les nomenclatures de produits, leur histoire, leur division économique en secteurs et branches, la répartition statistique des items et de certains résultats par branches.

3 J'esquisserai d'abord un bref historique, raccrochant la Grèce à l'activité internationale. Je développerai ensuite une analyse économique des transferts de valeur entre secteurs et branches et de leurs causes. Je livrerai enfin quelques hypothèses résultant de l'analyse statistique des nomenclatures industrielles dans cinq pays méditerranéens, comparée à celles de l'Union européenne (UE) à 27 et de l'Allemagne comme pays de référence. 


\section{Une brève histoire des nomenclatures}

4 Le tableau de Quesnay de 1758 est probablement une des premières nomenclatures d'activités économiques, car leur essor est plutôt contemporain de la Révolution industrielle. Pour simplifier, on distingue ici trois étapes: le milieu du $\mathrm{xIX}^{\mathrm{e}}$ siècle, l'entre-deux-guerres et l'après-guerre.

5 Le milieu du XIX ${ }^{\mathrm{e}}$ siècle est marqué par la Révolution industrielle qui touche à la fois les produits et la société. Le besoin d'enregistrer ces transformations est pressant: Adolphe Quételet, statisticien et sociologue, anime les Congrès internationaux de statistiques qui se réunissent de 1853 à 1876 et dont l'influence sur la création de commissions ou de bureaux statistiques dans les pays participants, et sur le lancement d'enquêtes industrielles, est déterminante. L'Institut International de Statistique (IIS) créé en 1885 prend le relais. De son côté, la Grèce délègue au Congrès statistique de Paris, en 1855, le Secrétaire général du ministère de l'Intérieur, Spyridon Spiliotakis. Le même est commissaire de la Grèce à l'Exposition universelle, la même année, à Paris. Alexandre Mansolas, dont on présente les chiffres, participe aux deux congrès statistiques de Saint-Pétersbourg en 1872 et de Budapest en 1876. Le tableau ci-dessous compare les « groupes » de produits grecs à ceux de l'Exposition universelle :

Tableau 1. Les groupes de produits grecs à l'Exposition universelle de 1867

\begin{tabular}{|c|c|c|c|c|c|c|c|}
\hline & & $\begin{array}{l}\text { Classes } \\
\text { Expo } \\
\text { Univ.1867 }\end{array}$ & $\begin{array}{l}\text { Classes } \\
\text { Mansolas }\end{array}$ & $\begin{array}{l}\text { Exposants } \\
\text { grecs }\end{array}$ & $\begin{array}{l}\text { Objets } \\
\text { exposés }\end{array}$ & $\begin{array}{l}\% \\
\text { exposants }\end{array}$ & $\begin{array}{l}\% \\
\text { objets }\end{array}$ \\
\hline Groupe 1 & CEuvres d'art & 5 & 5 & 18 & 44 & & \\
\hline Groupe 2 & $\begin{array}{l}\text { Matériel et } \\
\text { applications Des arts } \\
\text { libéraux }\end{array}$ & 8 & 5 & 23 & 76 & & \\
\hline Groupe 3 & $\begin{array}{l}\text { Meubles et objets } \\
\text { pour l'habitation }\end{array}$ & 13 & 5 & 18 & 38 & & \\
\hline Groupe 4 & $\begin{array}{l}\text { Vêtements (tissus } \\
\text { compris) et autres } \\
\text { Objets portés }\end{array}$ & 13 & 10 & 81 & 161 & 19,37 & 18,92 \\
\hline \multirow[t]{2}{*}{ Groupe 5} & $\begin{array}{l}\text { Produits bruts et } \\
\text { ouvrés } \\
\text { industries } \\
\text { extractives }\end{array}$ & 7 & 6 & 135 & 172 & & \\
\hline & $\begin{array}{l}\text { Dont agriculture non } \\
\text { alimentaire }\end{array}$ & 3 & 3 & 80 & 109 & 14,83 & 16,92 \\
\hline Groupe 6 & $\begin{array}{l}\text { Instruments et } \\
\text { procédés des arts } \\
\text { usuels }\end{array}$ & 21 & 5 & 7 & 10 & & \\
\hline
\end{tabular}




\begin{tabular}{|l|l|l|l|l|l|l|l|}
\hline Groupe 7 & $\begin{array}{l}\text { Aliments frais ou } \\
\text { conservés à divers } \\
\text { degrés } \\
\text { préparation }\end{array}$ & 7 & 6 & 291 & 350 & 50,79 & 41,13 \\
\hline Total & & 74 & 42 & 573 & 851 & 100 & 100 \\
\hline
\end{tabular}

Notons que les recensements de population de 1861 et de 1889 s'intéressent aux activités, mais moins aux produits qu'aux professions.

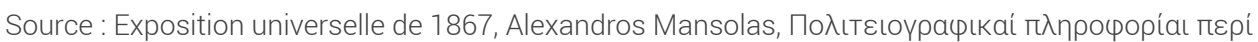

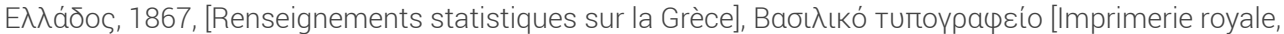
Athènes].

Tableau 2. Les professions dans le recensement grec de 1861

\begin{tabular}{|c|c|}
\hline Recensement de 1861 en Grèce & \\
\hline Propriétaires fonciers & 16122 \\
\hline Industriels & 32801 \\
\hline Agriculteurs & 147507 \\
\hline Bergers & 38953 \\
\hline Ouvriers & 19592 \\
\hline Domestiques hommes & 12651 \\
\hline Domestiques femmes & 7724 \\
\hline Voituriers et loueurs de chevaux & 2307 \\
\hline Artistes & 1346 \\
\hline Ecclésiastiques & 5102 \\
\hline Petits marchands & 9452 \\
\hline Gros négociants & 793 \\
\hline Marins de la marine royale & 510 \\
\hline Marins de la marine marchande & 19303 \\
\hline Employés et fonctionnaires & 3553 \\
\hline Employés des communes & 5199 \\
\hline Maîtres et professeurs & 1176 \\
\hline Avocats & 394 \\
\hline Journalistes & 68 \\
\hline Médecins & 398 \\
\hline Pharmaciens & 161 \\
\hline Sages femmes & 832 \\
\hline Étudiants garçons & 42680 \\
\hline Étudiants filles & 9035 \\
\hline
\end{tabular}


Total

377659

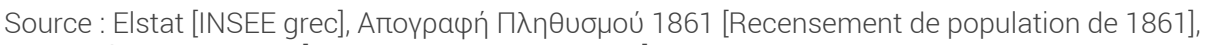

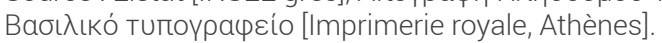

6 L'IIS et le Bureau international du Travail (BIT) ${ }^{2}$ sont les deux chevilles ouvrières de la normalisation de l'entre-deux-guerres. Le premier participe à la Commission d'étude de la Société des Nations (SDN) pour l'unification des méthodes de statistiques économiques. La Convention de 1928 charge un Comité d'experts de préparer une classification des industries qui aboutit, en 1938, à recommander une classification internationale par branches des activités économiques. Le BIT s'intéresse aux professions, mais recommande, par souci de rigueur, de caler ces classifications sur celles des activités et des produits.

7 En Grèce, Georges Chomatianos met en relation les travaux du recensement de 1907 et ceux des congrès statistiques internationaux et ceux de l'IIS. Le recensement de la population de 1928 cite les travaux du Comité d'experts de la SDN qui a développé « un projet de nomenclature des branches d'activité». D'ailleurs, la participation des statisticiens grecs à ces activités internationales mériterait sans doute un éclairage supplémentaire.

Enfin, en janvier 1947, la Commission de Statistique de l'ONU fait adopter l'International Standard Industrial Classification (ISIC). En 1970, Eurostat construit la Nomenclature des Activités économiques dans la Communauté européenne (NACE), harmonisée en 1988 avec l'ISIC et désormais obligatoire pour les États membres.

9 La Grèce adopte les nomenclatures internationales. Le recensement industriel de 1958 sous la direction de Pierre Kouvelis, se réfère à l'ISIC de 1948 :

La codification des branches d'activité a été opérée selon la Classification internationale type de toutes les branches d'activité économique, révisée par la Commission de Statistique de l'ONU, et dont les activités ont été subdivisées pour tenir compte des caractéristiques spéciales des « unités économiques » de la Grèce, afin que les données recueillies pussent être classées d'après leur cas d'espèce 3 .

Les nomenclatures d'activités, produits, professions, résultats de cette activité, sont toutes alignées sur les mêmes principes de comparabilité. Elles sont toutes construites sur des regroupements partiels par classes, branches et secteurs, affectés de codes décimaux. Elles prennent en compte à la fois le produit «naturel» et sa filière, le processus technologique et l'entreprise productrice. Telle est la dynamique historique qui sous-tend l'évolution des nomenclatures. Voyons la dynamique économique qui, elle aussi, entre en ligne de compte.

\section{Théorie économique et secteurs d'activité}

11 Les trois secteurs, primaire, secondaire et tertiaire n'ont pas seulement une valeur descriptive : on a essayé de théoriser la dynamique de passage d'un secteur à l'autre et, plus récemment, d'expliquer la désindustrialisation des années 1970. Dans cet esprit, une brève analyse quantitative des items des nomenclatures met en évidence la transformation profonde et des secteurs et des nomenclatures. 


\section{Les transferts intersectoriels.}

12 L'économie politique à la fin du XVII ${ }^{\mathrm{e}}$ siècle s'accompagne des premières nomenclatures, divisions analytiques de "la richesse des nations». Les physiocrates pensent que la seule activité économique est l'agriculture (plus largement les produits du sol, dont les mines) et que l'industrie consomme ses propres produits en les fabriquant et n'ajoute donc aucune valeur. William Petty, précurseur, note en $1682:$ « Il y a beaucoup plus à gagner par l'industrie que par l'agriculture, et beaucoup plus par le commerce que par l'industrie... $»^{4}$. Adam Smith fonde sur la division du travail l'enrichissement des nations et dessine une première esquisse des secteurs, calée sur l'offre ${ }^{5}$. Thomas Malthus, lui, s'intéresse à la distinction entre biens matériels et biens immatériels, mais pour rejeter ces derniers ${ }^{6}$. Jean-Baptiste Say, enfin, parmi ces précurseurs, considère que c'est l'utilité qui inspire l'activité économique, qu'il répartit entre les trois secteurs ${ }^{7}$. La valeur, l'utilité, et donc plutôt la demande, fondent l'économie, le reste est simple affaire de classification.

Il faut attendre Colin Clark et Allen Fisher en 1939 et 1940 pour une analyse dynamique des secteurs ${ }^{8}$. Leur définition repose sur la nature de leurs inputs, autrement dit de leur processus de production. Les glissements de l'activité de l'agriculture à l'industrie sont expliqués (Fisher) par l'élasticité des revenus à la demande, le tertiaire accueillant les produits, dont l'élasticité à la demande est la plus forte.

Jean Fourastié et Alfred Sauvy, en 1947, après la Seconde Guerre mondiale, montrent que c'est l'amélioration de la productivité, alimentée par l'innovation, qui constitue le moteur à la fois du déplacement de la demande (par la baisse des prix qu'elle engendre) et de l'emploi (en libérant une main-d'œuvre disponible pour d'autres secteurs) ${ }^{9}$. Alfred Sauvy parle de déversement. Ces analyses se réfèrent à la loi d'Ernst Engel de l'élasticité de la demande au revenu qui décroît des biens primaires aux biens tertiaires ${ }^{10}$.

\section{Désindustrialisation ou dématérialisation de l'industrie?}

La mutation profonde des activités dans les années 1970 est décrite souvent comme une désindustrialisation et une tertiarisation des économies. David Flacher et Jacques Pelletan montrent que les nomenclatures répondent mal aux mutations industrielles ${ }^{11}$ : la succession des trois Révolutions industrielles se caractérise par une multiplication considérable des produits, liée à l'évolution des besoins ${ }^{12}$. L'innovation, sa diffusion rapide dans l'économie, fait éclater le dispositif classificatoire. Mais ils soulignent également la «dématérialisation » progressive à la fois du produit et de la demande.

On a insisté sur le rôle de l'innovation, sur le déplacement de l'entreprise le long des secteurs à la recherche du profit et sur le flou consécutif des frontières (mines, agro-industrie, construction). Je voudrais insister sur la dématérialisation de la production et sur la modernité économique qui s'attache à l'immatérialité des biens. Jonathan Guershunny relève à ce sujet trois $\operatorname{cas}^{13}$ :

- Les biens culturels : la loi d'Engel, les goûts des ménages, l'innovation, se traduisent par une explosion de cette catégorie de biens, l'édition de contenus culturels sur tous supports - de moins en moins coûteux -, une production artistique déjà marchandisée depuis le début du siècle, l'importance croissante de la recherche et des logiciels. 
- Les services à l'industrie: le produit industriel se dématérialise, incorpore une valeur ajoutée intellectuelle de plus en plus grande, tels le design et les logiciels. L'entreprise externalise de nombreuses fonctions et les sous-traite, ainsi le marketing, la comptabilité, l'audit, la gestion de l'emploi, la gestion locative d'immeubles, de machines, de salariés.

- Enfin émerge une économie de self-service, d'autoproduction des ménages, de bricolage, liée à la montée du temps libre sous toutes ses formes, caractérisée par l'achat de biens d'équipement à prix relatif bas pour économiser un service. L'« autostegasi » en Grèce me paraît relever de ce cas $^{14}$.

\section{Analyses chiffrées des lignes de nomenclature}

La simple analyse quantitative des items des nomenclatures par secteur montre ces évolutions : approfondissement de la division du travail par multiplication du nombre des items dans le temps, dans l'entre-deux-guerres et surtout l'après Seconde Guerre mondiale. En revanche, la fluidité des secteurs et les déversements de l'agriculture vers l'industrie, puis les services ne sont pas vraiment avérés. Mais une analyse plus fine par branche confirme le « déversement » de l'industrie vers les services à l'industrie et les produits culturels.

Les tableaux 3 et 4 , en annexe, concernent la Grèce : curieusement, dans ce pays, la description des professions met l'accent sur les professions libérales, puis, lentement, sur les professions industrielles. L'industrie, à partir de 1907, trouve sa place qui croît jusqu'en 1928. La décroissance des services en 1928 est une anomalie. Après la Seconde Guerre mondiale, l'industrie, comme activité, maintient sa place jusqu'en 2002, avant de décroître au profit des services.

Les tableaux 5, 6 et 7 décrivent successivement les nomenclatures d'activités ISIC, de produits CPC et CPA, de professions (STP et ISCO). Au niveau des activités, il y a bien montée, puis décroissance de l'industrie, et inversement, des services. Au niveau des produits, en revanche, l'inflation des items industriels plus faciles à identifier empêche les services de trouver leur place : ce n'est qu'en 2008 que le toilettage se fait. Au niveau des professions, la décroissance de l'industrie est vive et se fait au profit des services: la sensibilité de la statistique aux structures sociales est plus grande. Le tableau 6 détaille les services. Le tableau 9 aussi, mais il décrit la difficulté d'un pays du Sud, la Grèce, à rentrer dans le cadre général de l'Union européenne à travers l'onomatologia ${ }^{15}$ de 2008. Celle-ci spécifie les caractéristiques grecques: le commerce de détail (à huit chiffres il est vrai) y représente 1036 items, contre 202 dans le CPA de l'UE à six chiffres et le commerce de gros 1635 contre 106. Inversement, l'industrie est nettement mieux représentée dans le CPA que dans l'onomatologia. C'est le premier indice d'inadaptation des nomenclatures européennes aux réalités méditerranéennes.

\section{Nomenclatures industrielles en Méditerranée}

20 Ce travail sur les nomenclatures nous permet, à partir des résultats observés sur les pays méditerranéens qui nous intéressent, de tenter de vérifier l'existence d'un profil méditerranéen comparé à l'ensemble de l'UE à 27 et à titre de référence, à l'Allemagne.

21 J'ai utilisé deux cadres statistiques homogènes, les tableaux emplois ressources des comptabilités nationales et les enquêtes Prodcom (productions communautaires). 
Voir Tableaux emplois ressources des comptabilités nationales (Tableaux 10 et 11 en annexe)

La moyenne des quatre pays méditerranéens (hors Turquie) comparée aux valeurs correspondantes de l'Allemagne met en évidence, au niveau des PIB, la part de l'agriculture et celle des services, nettement supérieures, et inversement celle de l'industrie, nettement inférieure. Toutefois, l'Italie et l'Espagne présentent un profil industriel plus proche de celui de l'Allemagne que de ceux de la France ou de la Grèce et, pour l'Italie notamment, dans le cas des machines. Pour l'Espagne cependant, il faudrait corriger l'anomalie que constitue la construction: la part de l'industrie rejoindrait la moyenne européenne. En revanche, la moyenne des quatre pays méditerranéens ne s'écarte de celle de l'UE 27 que pour affirmer un profil inverse où la part de l'industrie est plus élevée, et celle des services, moindre. Indices de développement plus que de localisation : Chastagnaret a sans doute raison.

L'analyse fine des services fait apparaitre un profil sinon méditerranéen, en tout cas spécifique. Le rapprochement Grèce-France est très remarquable, si l'on considère l'administration publique, mais la moyenne des quatre ne diffère pas de celle de l'UE, et à peine de celle de l'Allemagne. En revanche, services immobiliers, produits culturels, commerce - transports - télécoms distinguent nettement les quatre pays méditerranéens de la moyenne européenne, et plus encore de l'Allemagne. Si l'on peut hésiter au niveau des PIB, on ne le peut pas au niveau des Valeurs Ajoutées et des Output. Le cas français des services à l'industrie ( $14,2 \%$ de la VA) mériterait une étude à part, qui corrige, en partie, la faiblesse de la part de l'industrie commune aux trois autres pays du Sud. Notons le profil très spécifique de la Grèce, intégrant une vision particulière du commerce et le rôle stratégique des transports et des communications (presse, audiovisuel, télécom). Depuis 2010, l'activité du port du Pirée connaît la plus forte croissance en Europe, ce qui renforce la part de ce poste.

\section{Tableaux prodcom}

5 Les limites inhérentes aux TER n'existent pas ici. Les produits industriels sont classés par branches, mais on dispose du détail à l'intérieur des branches. De plus, Eurostat dispose des enquêtes par pays jusqu'en 2014. La seule limite est qu'ils ne comportent ni les produits agricoles ni les biens immatériels et services.

26 La première observation (Tableau 12) concerne le remplissage des cases par chacun. Les zones non renseignées correspondent à des absences de production. La Grèce, et à un moindre degré, la Turquie, ont un taux élevé de lignes non renseignées, l'Allemagne ayant le taux le plus bas. France, Italie, et Espagne ont des taux très bas, proches de ceux de l'Allemagne. La confidentialité n'est pas forcément un trait méditerranéen, l'Allemagne ayant un taux plus élevé que la Grèce, et l'Italie ayant le taux le plus bas.

La deuxième observation est relative au nombre d'items par branche: les industries traditionnelles, y compris l'agroalimentaire, ne représentent que le tiers des lignes, alors que la métallurgie et les machines et matériels en représentent $48 \%$, et la chimie et ses dérivés $19 \%$. On rejoint la remarque ci-dessus sur l'onomatologia grecque : la nomenclature est faite pour des pays dont le profil industriel est bien précis, et comme par hasard, proche du profil allemand. Là encore, la Méditerranée ne rentre pas bien dans la focale. 

méditerranéen dont les valeurs moyennes occupent une place symétriquement inverse de celle de l'Allemagne par rapport à la moyenne européenne. Le tableau 13 expose les parts de branches industrielles dans le total Prodcom, dans les quatre pays méditerranéens, en Allemagne et dans l'UE 27. En mode simplifié, on a regroupé ces quatre pays, l'Allemagne et l'UE 27 pour les IAA (Industries agroalimentaires), la chimie (plus plastiques et pharmacie) et l'industrie "lourde" (NACE, 24 à 30). Il nous semble bien que les 4 pays méditerranéens partagent des caractéristiques nettement différentes de celles de l'Allemagne. Le poids des IAA leur est commun, et, même si l'Italie détonne, son "régime alimentaire" reste bien caractérisé. Les industries «traditionnelles» comme le textile, le bois, le papier, les matériaux de construction ont en général un poids particulier (encore l'Espagne et la Grèce ont-elles un secteur immobilier en plein effondrement). L'Italie, qui se rapprocherait plutôt de l'Allemagne, s'en distingue nettement au niveau des industries "traditionnelles ", qui représentent $15 \%$ de sa production, contre $10 \%$ en Allemagne, indiquant l'importance de la Troisième Italie. Inversement, les industries « lourdes » pèsent en Allemagne d'un poids exceptionnel. Enfin, la chimie et ses dérivés, sauf en Italie, ont un poids assez proche, ce qui montre que la Méditerranée n'exclut pas par nature ce type d'activités.

Le tableau 14 reprend ces mêmes données Prodcom par pays, pour extraire le poids des branches d'un pays lorsqu'elles y représentent au moins $10 \%$ de la production de l'UE 27. C'est une sorte de mesure de la concentration de certaines productions dans certains pays. C'est aussi une invitation à regarder de plus près encore ces productions : les mines en Grèce, bauxite, bentonite et perlite, sont une spécialité de dimension européenne, encore plus nette si l'on y ajoute les produits métalliques (alumine et aluminium et tubes de cuivre), comme aussi certaines fabrications textiles spécialisées (fourrures, fils de coton peigné pour tapis de sol, sacs plastiques, filets à maille noués et cordages en textile naturel ou synthétique). L'Espagne et l'Italie se distinguent aussi dans ces branches traditionnelles du textile, de l'habillement et du cuir (on peut y ajouter l'ameublement). L'originalité des profils est donc très prononcée et cette sélection l'amplifie. À la détermination géographique, il nous semble cependant nécessaire d'ajouter une détermination historique: ancienneté de l'industrialisation, aléas politiques, et peut-être une détermination culturelle, manière de répondre aux défis du siècle.

\section{Conclusions}

- Les nomenclatures sont filles des Lumières (transparence, universalité).

- L'industrie a changé de forme et avec elle, sans doute, l'emploi, l'organisation du travail et les formes de conflits sociaux.

- Le profil méditerranéen existe, mais il faut l'apprécier au niveau détaillé des productions industrielles; ce n'est qu'à ce niveau fin qu'il subvertit les nomenclatures et dénonce le regard qu'elles portent sur lui, mais aussi se révèle.

- On pressent que ces formes d'industries propres à ces pays méditerranéens sont liées à la localisation des matières premières, mais qu'elles induisent des formes d'emploi elles aussi «méditerranéennes». À vrai dire, ces produits dessinent une anthropologie que l'exploration des comportements des hommes approfondirait, révélant la " méditerranéité » qui nous échappe encore.

Cahiers balkaniques, 45 | 2018 


\section{Annexes}

Tableau 3. Nomenclatures professionnelles dans les recensements de population en Grèce de 1861 à 1928.

\begin{tabular}{|l|l|l|l|l|}
\hline \multicolumn{5}{|c|}{ Répartition des items par secteur dans les recensements Elstat } \\
\hline & 1861 & 1907 & 1920 & $1928^{*}$ \\
\hline Agriculture & 2 & 10 & 20 & 23 \\
\hline Industrie & 2 & 87 & 113 & 137 \\
\hline Services & 20 & 100 & 114 & 94 \\
\hline & 24 & 197 & 247 & 254 \\
\hline Répartition des items par secteur dans les recensements Elstat en \% \\
\hline Agriculture & 8,33 & 5,08 & 8,10 & 9,06 \\
\hline Industrie & 8,33 & 44,16 & 45,75 & 53,94 \\
\hline Services & 83,33 & 50,76 & 46,15 & 37,01 \\
\hline & 100 & 100 & 100 & 100 \\
\hline
\end{tabular}

Tableau 4. Nomenclatures d'activité en Grèce après la Seconde Guerre mondiale d'après les recensements

\begin{tabular}{|l|l|l|l|l|l|l|l|}
\hline Items & 1951 & 1960 & 1971 & 1980 & Stakod91 & Stakod32002 & Stakod82008 \\
\hline Agriculture & 23 & 40 & 37 & 39 & 23 & 34 & 39 \\
\hline Industrie & 210 & 391 & 365 & 396 & 289 & 294 & 284 \\
\hline Services & 299 & 325 & 280 & 324 & 224 & 236 & 291 \\
\hline & & & & & & & \\
\hline$\%$ & & & & & & & \\
\hline Agriculture & 4,32 & 5,29 & 5,43 & 5,14 & 4,29 & 6,03 & 6,35 \\
\hline Industrie & 39,47 & 51,72 & 53,52 & 52,17 & 53,92 & 52,13 & 46,25 \\
\hline Services & 56,20 & 42,99 & 41,06 & 42,69 & 41,79 & 41,84 & 47,39 \\
\hline & 100 & 100 & 100 & 100 & 100 & 100 & 100 \\
\hline
\end{tabular}

Tableau 5. Évolution des items des nomenclatures CITI (Classification Internationale Type par Industrie) (ISIS) de I'ONU

Classification Internationale Type par Industrie. En nombre et en \%

\begin{tabular}{|l|l|l|l|l|l|}
\hline & $\mathbf{1 9 4 8}$ & Rév 1 1958 & Rév 2 1968 & Rév 3 1989 & Rév 42008 \\
\hline CITI nombres & 1948 & 1958 & 1968 & 1989 & 2008 \\
\hline Agriculture & 5 & 8 & 7 & 10 & 36 \\
\hline Industrie & 40 & 76 & 94 & 141 & 173 \\
\hline
\end{tabular}




\begin{tabular}{|l|l|l|l|l|l|}
\hline Services & 35 & 40 & 59 & 134 & 219 \\
\hline total & 80 & 124 & 160 & 285 & 430 \\
\hline CITI en \% & & & & & \\
\hline Agriculture & 6,25 & 6,45 & 4,38 & 3,51 & 8,84 \\
\hline Industrie & 50 & 61,29 & 58,75 & 49,47 & 40,23 \\
\hline Services & 43,75 & 32,26 & 36,88 & 47,02 & 50,93 \\
\hline Total & 100 & 100 & 100 & 100 & 100 \\
\hline
\end{tabular}

Tableau 6. Nomenclatures de produits CPC (ONU) et CPA (Union européenne)

\begin{tabular}{|l|l|l|l|l|l|l|}
\hline & CPC 1989 & CPC 2002 & CPC2008 & CPA 1993 & CPA 2002 & CPA 2008 \\
\hline Items & & & & & & \\
\hline Agriculture & 80 & 83 & 113 & 94 & 96 & 204 \\
\hline Industrie & 1145 & 1122 & 1379 & 1541 & 1813 & 2006 \\
\hline Services & 586 & 893 & 814 & 670 & 699 & 1008 \\
\hline Total & 1811 & 2098 & 2306 & 2305 & 2608 & 3218 \\
\hline \% & & & & & & \\
\hline Agriculture & 4,42 & 3,96 & 4,90 & 4,08 & 3,68 & 6,34 \\
\hline Industrie & 63,22 & 53,48 & 59,80 & 66,85 & 69,52 & 62,34 \\
\hline Services & 32,36 & 42,56 & 35,30 & 29,07 & 26,80 & 31,22 \\
\hline Total & 100 & 100 & 100 & 100 & 100 & 100 \\
\hline
\end{tabular}

Tableau 7. Nomenclatures professionnelles en Grèce et internationales (ISCO)

\begin{tabular}{|l|l|l|l|l|l|l|}
\hline Items & $\mathbf{1 9 7 3} \mathbf{3}$ chiffres & $\mathbf{1 9 9 2} \mathbf{4}$ chiffres & ISCO 1958 & ISCO 1968 & ISCO 1998 & ISCO 2008 \\
\hline Agriculture & 43 & 40 & 5 & 16 & 20 & 26 \\
\hline Industrie & 129 & 145 & 108 & 119 & 134 & 100 \\
\hline Services & 135 & 216 & 202 & 285 & 464 & 436 \\
\hline Total & & & & & & \\
\hline$\%$ & & & & & & \\
\hline Agriculture & 14,01 & 9,98 & 2,48 & 5,61 & 4,31 & 5,96 \\
\hline Industrie & 42,02 & 36,16 & 53,47 & 41,75 & 28,88 & 22,94 \\
\hline services & 43,97 & 53,87 & 44,06 & 52,63 & 66,81 & 71,10 \\
\hline Total & 100 & 100 & 100 & 100 & 100 & 100 \\
\hline
\end{tabular}


Tableau 8. Détail des Services dans les nomenclatures d'activités grecques (Stakod) et ONU

\begin{tabular}{|l|l|l|l|l|l|l|}
\hline Services & Stakod 91 & Stakod 3 2002 & Stakod 8 2008 & ISIC 1968 & ISIC 1989 & ISIC 2008 \\
\hline Transports & 21 & 21 & 23 & 5 & 17 & 20 \\
\hline Services industrie & 30 & 32 & 56 & 4 & 25 & 41 \\
\hline Informatique & 6 & 7 & 8 & 0 & 6 & 6 \\
\hline Produits culturels & 16 & 17 & 32 & 3 & 11 & 27 \\
\hline Services personne & 6 & 11 & 16 & 4 & 7 & 15 \\
\hline
\end{tabular}

Tableau 9. Items dans 2 nomenclatures de produits CPA (Union européenne) et Grèce

\begin{tabular}{|l|l|l|l|l|}
\hline Items & CPA 2008 & Onomatologia 2008 & $\%$ CPA & $\%$ Grèce \\
\hline Agriculture & 205 & 190 & 6 & 4 \\
\hline Industrie & 1921 & 555 & 59 & 11 \\
\hline Services & 1147 & 4087 & 35 & 85 \\
\hline Total & 3273 & 4833 & 100 & 100 \\
\hline & \multicolumn{2}{|l}{ Source Elstat/Ramon 8 chiffres } & & \\
\hline
\end{tabular}

Tableau 10. PIB, en \% du PIB total, de quelques pays de l'UE et de la Turquie, par secteur (TER Eurostat), en 2010

\begin{tabular}{|l|l|l|l|l|l|l|l|l|}
\hline PIB & Grèce & Espagne & Italie & France & $\begin{array}{l}\text { Moyenne des } \\
4\end{array}$ & UE 27 & Allemagne & Turquie \\
\hline Agriculture & 2,94 & 2,14 & 1,83 & 1,67 & 2,15 & 1,68 & 0,80 & 10,52 \\
\hline Industrie & 23,56 & 31,47 & 29,72 & 24,11 & 27,21 & 25,34 & 34,75 & 33,74 \\
\hline Services & 73,50 & 66,40 & 68,46 & 74,22 & 70,65 & 72,98 & 64,45 & 55,74 \\
\hline & & & & & & & & \\
\hline PIB & & & & & & & & \\
\hline Agriculture+ IAA & 8,39 & 5,62 & 4,87 & 4,62 & 5,87 & 3,73 & 3,79 & 15,65 \\
\hline $\begin{array}{l}\text { Travail métaux et } \\
\text { machines }\end{array}$ & 2,80 & 4,69 & 7,42 & 4,54 & 4,87 & 6,83 & 12,33 & 5,64 \\
\hline
\end{tabular}

Tableau 11. Étude de profils Services (VA Eurostat 2010)

\begin{tabular}{|l|l|l|l|}
\hline Étude de profil VA & Allemagne & Quatre pays & UE 27 \\
\hline Administration & 18,32 & 19,57 & 19,41 \\
\hline Produits culturels & 4,39 & 7,83 & 5,54 \\
\hline Commerce, transp. Télécom. & 12,07 & 17,12 & 15,54 \\
\hline
\end{tabular}




\begin{tabular}{|l|l|l|l|}
\hline Immobilier & 16,54 & 18,87 & 16,77 \\
\hline Services industrie & 12,57 & 9,88 & 11,9 \\
\hline & & & \\
\hline Étude de profil output & Allemagne & Quatre pays & UE 27 \\
\hline Administration & 12,82 & 13,4 & 13,47 \\
\hline Produits culturels & 4,16 & 7,1 & 5,25 \\
\hline Commerce, transp. Télécom. & 12,1 & 17,2 & 15,15 \\
\hline Immobilier & 12,47 & 15,5 & 14,34 \\
\hline Services industrie & 10,25 & 9,5 & 10,46 \\
\hline
\end{tabular}

Tableau 12. Enquêtes Prodcom : taux de remplissage des 3814 lignes de produits en 2014

\begin{tabular}{|l|l|l|l|l|l|l|}
\hline & Grèce & France & Italie & Espagne & Allemagne & Turquie \\
\hline Non renseigné & 57 & 10 & 10 & 12 & 7 & 24 \\
\hline Confidentiel & 23 & 38 & 18 & 26 & 29 & 40 \\
\hline Renseigné & 20 & 52 & 71 & 62 & 65 & 36 \\
\hline Total & 100 & 100 & 100 & 100 & 100 & 100 \\
\hline
\end{tabular}

Tableau 13. Part de certaines branches dans le total Prodcom 2014 par pays

\begin{tabular}{|l|l|l|l|l|l|l|}
\hline & Grèce & Espagne & Italie & France & Allemagne & UE 27 \\
\hline Industries agroalimentaires & 47,53 & 32,03 & 17,58 & 28,45 & 14,20 & 19,32 \\
\hline Textile habillement cuir & 3,56 & 3,30 & 6,81 & 1,61 & 0,97 & 2,64 \\
\hline Bois ameublement & 1,47 & 2,31 & 4,06 & 2,61 & 2,98 & 3,20 \\
\hline Papier presse & 5,41 & 4,53 & 4,75 & 4,05 & 3,69 & 4,21 \\
\hline Chimie pharmacie plastiques & 15,84 & 14,91 & 13,04 & 17,21 & 16,86 & 16,56 \\
\hline Produits non métalliques & 5,18 & 3,63 & 3,88 & 3,70 & 2,39 & 3,06 \\
\hline Métallurgie de base & 10,90 & 5,71 & 7,57 & 2,58 & 6,99 & 6,99 \\
\hline Prod. électr. électron. Mach-mat transp. & 7,35 & 29,23 & 36,78 & 31,54 & 46,43 & 38,44 \\
\hline Réparation installation & 0,90 & 3,00 & 2,96 & 6,09 & 3,38 & 2,98 \\
\hline
\end{tabular}

Ou en simplifiant: Industries lourdes regroupe Métallurgie de base, produits électriques, électroniques, machines et matériels de transport

Tableau 14. Part des branches dans Prodcom 2014, par pays, lorsqu'elles y représentent plus de $10 \%$ du total UE

\begin{tabular}{|l|l|l|l|l|l|}
\hline Plus de $10 \%$ & Grèce & Espagne & Italie & France & Allemagne \\
\hline Mines & 14,3 & 1,8 & 1 & 1,3 & 0,6 \\
\hline
\end{tabular}




\begin{tabular}{|l|l|l|l|l|l|}
\hline IAA & 38,1 & 21,5 & 9,1 & 18 & 9,1 \\
\hline Textile & 14,3 & 6,7 & 8 & 3,8 & 2,5 \\
\hline Habillement & 4,8 & 6,7 & 5,4 & 1,1 & 0,4 \\
\hline Cuir & 4,8 & 2,5 & 2 & 1,1 & 0,2 \\
\hline Bois & 0 & 2,1 & 1,7 & 2,1 & 1,3 \\
\hline Papier & 4,8 & 3 & 2,7 & 3 & 2,6 \\
\hline Impression et autres media & 0 & 1,1 & 1,1 & 1,3 & 1 \\
\hline Chimie & 0 & 15,3 & 7,8 & 12 & 13,3 \\
\hline Pharmacie & 0 & 1,1 & 0,8 & 1,2 & 1,1 \\
\hline Caoutchouc, Mat. plastiques & 0 & 3,1 & 4,1 & 4,6 & 4,8 \\
\hline Autres prod. non métall. & 4,8 & 4,9 & 4,2 & 4,8 & 3,8 \\
\hline Métallurgie de base & 9,5 & 4,8 & 6,7 & 3 & 6,7 \\
\hline Produits métalliques & 0 & 7,7 & 10,3 & 10,2 & 10,5 \\
\hline Électronique, optique & 0 & 1,1 & 2,4 & 4,7 & 7,1 \\
\hline Mat. électrique & 4,8 & 2,5 & 5,7 & 5,7 & 6,7 \\
\hline Machines, équipement & 0 & 6,6 & 17 & 10,6 & 19,1 \\
\hline Véhicules à moteur & 0 & 1,1 & 1 & 1,6 & 1,6 \\
\hline Autres transports & 0 & 1,1 & 1,5 & 1,2 & 1,2 \\
\hline Ameublement & 0 & 0,7 & 1,2 & 0,8 & 0,9 \\
\hline Divers industries & 0 & 2,1 & 3,7 & 3,7 & 3,2 \\
\hline Réparation, installation & 0 & 2,4 & 2,4 & 4,1 & 2,3 \\
\hline Total & 100 & 100 & 100 & 100 & 100 \\
\hline
\end{tabular}

\section{BIBLIOGRAPHIE}

\section{Monographies et articles de revues}

CAhuc Pierre \& Debonneuil Michèle, 2004, «Productivité et emploi dans le tertiaire », Centre d'Analyse économique, La Documentation française $\mathrm{n}^{\circ}$ 5, Paris, $249 \mathrm{p}$.

CAIRE Guy, 1965, « Un précurseur négligé : William Petty, ou l'approche systématique du développement économique », Revue économique, vol. 16, n5 5, p. 734-776.

CARON François, 1997, Les deux révolutions industrielles du XXe siècle, Albin Michel, Paris, 528 p. 
CHASTAGNARET Gérard, 1997, «L'industrie en Méditerranée : une histoire en construction », Méditerranée, Tome 87, 3-4, Industries en Méditerranée de la marginalisation à la mondialisation. p. 5-12.

CLARK Colin, 1940, The Conditions of Progress and Security, Macmillan, London, 720 p.

DEBONNEUIL Michèle, 2007, L'espoir économique : vers la révolution du quaternaire, François Bourin, Paris.

DEBONNEUIL Michèle, 2010, L'économie quaternaire : une croissance durable à construire, Centre d'analyse stratégique. URL : http://archives.strategie.gouv.fr/cas/system/files/cb992032d01.pdf, consulté le 7 avril 2014.

Delaunay Jean-Claude \& GADREY Jean, 1987, Les enjeux de la société de services, Presses de la FNSP, Paris, $333 \mathrm{p}$.

DESROSIÈRES Alain, 1972, « Un découpage de l'industrie en trois secteurs », Économie et Statistique, $n^{\circ} 40$, p. 25-39.

DESROSIÈRES Alain, 1985, « Histoires de formes : statistiques et sciences sociales avant 1940 », Revue française de sociologie, 26-2, p. 277-310.

DESROSIÈRES Alain, 1977. "Éléments pour l'histoire des nomenclatures socioprofessionnelles ", Pour une histoire de la statistique, tome I, INSEE, Paris, p. 155-231.

DUMONTIER Jacques, 1965, «Généralisation de la loi des trois secteurs », Revue économique, Vol. 16, $\mathrm{n}^{\circ}$ 6, p. 974-978.

FISCHER Allan, 1935, The Clash of Progress and Security, Macmillan, London.

FiSHER Allan, 1939, "Production, primary, secondary and tertiary" Economic Record 15.1, p. 24-38.

FlaCher David \& PelLETAN Jacques, 2007, « Le concept d'industrie et sa mesure : origine, limites et perspectives. Une application à l'étude des mutations industrielles ", Économie et Statistique, $n^{\circ} 405 / 406$, p. 13-46.

FourASTIÉ Jean, 1963, Le grand espoir du XXe siècle, Gallimard, Paris, Collection « Idées », $\mathrm{n}^{\circ} 20$.

FOURASTIÉ Jean, 1952, La Productivité, PUF, Paris.

GADREY Jean, 2002, « Une économie de services », Les Cahiers français, $\mathrm{n}^{\circ}$ 311, p. 1-27.

GERSHUNY Jonathan, 1983, Social Innovation and the Division of Labor, Oxford University Press, Oxford, $191 \mathrm{p}$.

GERSHUNY Jonathan, 1978, After Industrial Society, the Emerging Self-Service Economy, Macmillan, London, $192 \mathrm{p}$.

GUILBERT Bernard, LAGANIER Jean \& VolLE Michel, 1971, « Essai sur les nomenclatures industrielles ", Économie et statistique, $\mathrm{n}^{\circ}$ 20, p. 23-36.

LAINÉ Frédéric, 1999, « Logiques sectorielles et nomenclatures d'activités », Économie et Statistique, $n^{\circ} 323$, p. 95-113.

LANDES David S., 1969, Unbound Prometheus. Technological change and industrial development in Western Europe from 1750 to the present, Cambridge University Press, Cambridge, $590 \mathrm{p}$.

LESTANG Philippe, 1973, «Le nouveau système français des nomenclatures d'activités et de produits ",Économie et statistique, $\mathrm{n}^{\circ}$ 51. p. 55-56.

Malthus Thomas, (1820), 1969, Principes d'économie politique, Calmann Levy, Paris. 
PALAIRET Michel, 1985, « Désindustrialisation à la périphérie : études sur la région des Balkans au $\mathrm{XIX}{ }^{\mathrm{e}}$ siècle ». Histoire, économie et société, $4^{\mathrm{e}}$ année, $\mathrm{n}^{\circ}$ 2. p. 253-274.

PetTy William, 1905 (1682), "Political Arithmetic" in Les Euvres économiques de Sir William Petty, Giard et Brière, Paris.

QUÉTELET Adolphe, 1873, Congrès international de statistique de Bruxelles 1853, Bruxelles , Imprimerie royale de Belgique, Bruxelles, URL : http://gallica.bnf.fr/ark:/12148/bpt6k6540041f, consulté le 2 mai 2015.

SAUVY Alfred, 1980, La machine et le chômage, Dunod, Paris, 336 p.

SAY Jean Baptiste, 1803, Traité d'économie politique ou simple exposition de la manière dont se forment, se distribuent et se composent les richesses, 1ère éd., Crapelet, Paris.

SмIтH Adam, 2012 (1776), Wealth of Nations, Wordsworth Classics of World Literature, Worsworth editions ltd, London, $1008 \mathrm{p}$.

TOFfLER Alvin, 1980, La troisième vague, Denoël, Paris, 623 p.

Volle Michel, 1982, Histoire de la statistique industrielle, Economica, Paris, 302 p.

ZARKA Claude, 1965, «Intensité du progrès technique et classification des activités économiques en trois secteurs ", Revue économique, vol. 16, n² 2, p. 199-211.

\section{Autres sources}

Archives statistiques françaises

Enquête sur les manufactures de Colbert, 1669.

Enquête sur l'industrie, 1861.

INSEE : guide des nomenclatures.

La France économique : statistique raisonnée et comparative par Alfred de Foville, 1887.

La statistique avant l'I.N.S.E.E., 1971, Économie et statistique, $\mathrm{n}^{\circ}$ 24, juin. p. 14-16.

Nomenclature de 1913 des industries et professions.

Statistique générale de la France : « Industrie 1847 ».

Statistique générale de la France : « Industrie, enquête de 1861-1865 », Paris, 1873.

Statistique générale de la France : « Recensement de 1896. Industrie ».

Tableau de Quesnay, 1758.

Tableau des principales industries de la France de Tolosan, 1788.

Tableau de l'état physique et moral des ouvriers de Villermé, 1840.

\section{NOTES}

1. «[... ] lors de la grande Exposition de Londres, ce bazar universel où toutes les parties du monde civilisé sont venues étaler les merveilles de leur art et de leur industrie, ce n'était pas seulement la confusion des langues qui faisait obstacle à l'échange des idées ; c'était surtout l'insuffisance où l'on était de comparer tant de choses et de ramener à 
une même appréciation les forces et les richesses de tant de nations ». "Il devenait indispensable d'employer les mêmes méthodes et d'observer les mêmes instruments, portant les mêmes échelles et gradués de manière homogène ». QUÉTELET, 1873, p. 7.

2. Albert Thomas, premier directeur du BIT participe en 1914, au ministère de l'Armement, à l'organisation de la production industrielle en temps de guerre, puis il y est comme sous-secrétaire d'État, puis comme ministre. François Simiand, l'historien des fluctuations économiques, l'assiste au ministère, et Lucien March, précédemment responsable de la Statistique à l'office du travail, est alors directeur de la Statistique générale de la France.

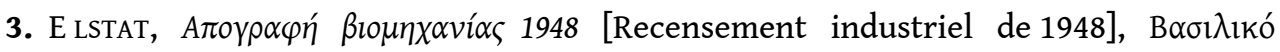

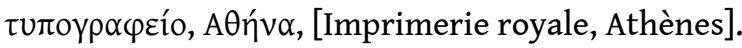

4. William PETTY, 1905.

5. SмIтH, 2012, p. 354: "Of the different employment of capital: A capital may be employed in four different ways: first in procuring the rude produce [primaire], or secondly in manufacturing and preparing the rude produce [secondaire], or thirdly in transporting either the rude or the manufactures produce or lastly in dividing portions of either..."[tertiaire, transport et commerce]

6. Malthus, 1969, Chapitre 1, Des définitions de la richesse : «La ligne qu'il est le plus naturel et le plus utile de tracer nettement est celle qui sépare les objets matériels des objets immatériels ».

7. SAY, 1803. Livre premier, chapitre II : « Lorsqu'elle (notre industrie) se borne à les (les produits de la nature) recueillir des mains de la nature, on la nomme industrie agricole, ou simplement agriculture. Lorsqu'elle sépare, mélange, façonne les produits de la nature, pour les approprier à nos besoins, on la nomme industrie manufacturière. Lorsqu'elle met à notre portée les objets de nos besoins, qui n'y seraient pas sans cela, on la nomme industrie commerciale, ou simplement commerce». "Toutes (les activités) donnent une utilité à ce qui n'en avait point ».

8. FISHER, 1939, p. 24-38, CLARK, 1940.

9. FoURASTIÉ, 1952, SAUVY, 1981.

10. ENGEL, créateur de l'Office saxon des Statistiques en 1850, décrit en 1857 le résultat de son analyse des budgets ouvriers.

11. Flacher \& Pelletan, 2007, p. 13-46, « Le nom même d'industrie, tel qu'il a été façonné par l'histoire, plus qu'un simple découpage statistique, doit beaucoup au modèle de civilisation de la révolution industrielle occidentale, qui repose sur les innovations techniques et organisationnelles dans la production, sur les nouveaux produits, mais aussi sur les tensions et les luttes sociales ».

12. CARON, 1997.

13. GERSHUNY, 1978.

14. Autoconstruction (par les ménages et non par des professionnels).

15. «Dénomination » des produits soumis à tarifs douaniers. 


\section{RÉSUMÉS}

Vincent Gouzi s'est penché sur les nomenclatures industrielles en Grèce et, à travers leur histoire et leur structure, sur les variations du champ du secteur secondaire et les limites de comparabilité.

Les nomenclatures industrielles ont profondément évolué en Grèce comme en France ; depuis le milieu du XIX ${ }^{e}$ siècle, elles ont été profondément influencées par les institutions internationales. Les moteurs de cette évolution sont l'innovation, les conceptions de l'organisation industrielle, et le besoin de comparabilité.

Ce papier a pour but de rappeler en premier lieu la théorie économique des nomenclatures (notamment leur structure évolutive entre les trois secteurs d'activités) ainsi que l'histoire des nomenclatures industrielles. En second lieu, il tente de définir une spécificité méditerranéenne à travers les items constitutifs de différentes nomenclatures, l'analyse des tableaux d'emplois et ressources de la comptabilité nationale des cinq pays étudiés et l'analyse de leurs productions industrielles.

Vincent Gouzi bend over industrial classifications in Greece, and, through their history and their structure, over the variations of the field of the secondary sector and the limits of comparability. The industrial classifications profoundly evolved in Greece as in France since the middle of the $19^{\text {th }}$ century, profoundly influenced by the international institutions. The boosters of this evolution are the innovation, the conceptions of the industrial organization and the needs for comparability.

This paper aims first at reminding the economic theory of the classification of activities (in particular their evolutionary structure between the three economic sectors) as well as the history of the industrial classifications. Secondly, it endeavours to define a Mediterranean specificity through the constituent items of different classifications, the analysis of the input-output tables of the national accountings of the five countries and of their industrial productions.

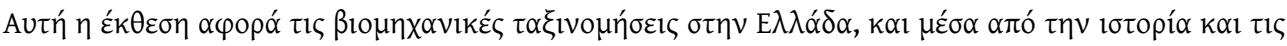

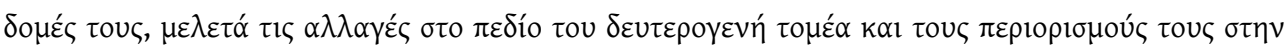

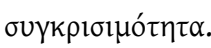

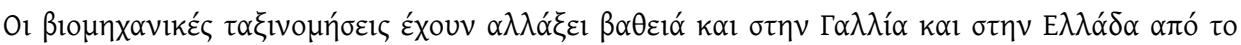

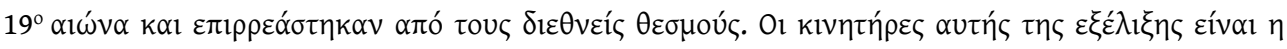

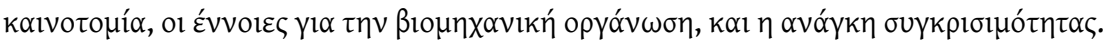

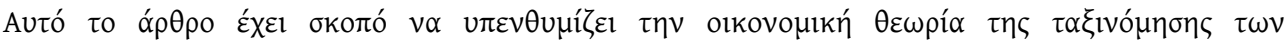

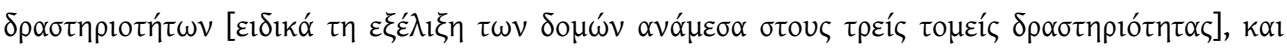

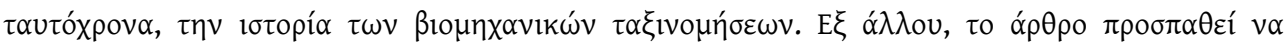

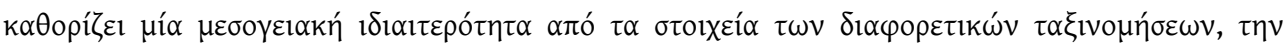

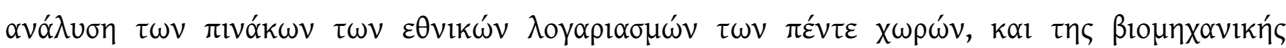
$\pi \alpha \rho \alpha \gamma \omega \gamma \eta \dot{s}$ тоus. 


\section{INDEX}

motsclestr terminoloji, sanayi, kategori, şube, sektör, Yunanistan, Akdeniz özgüllüğü, sayım, ekonomik teoriler, sanayisizleşme, çağdaş dönem, Akdeniz, sosyal tarih, ekonomik tarih Keywords : classification, industry, category, branch, sector, Greece, Mediterranean specificity, census, economic theories, des-industrialization, contemporary period, social history, economic history

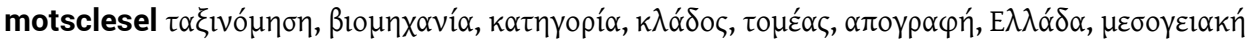

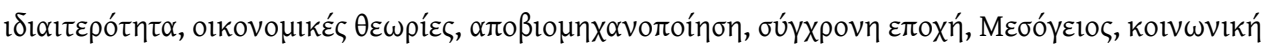

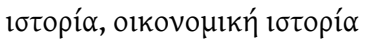

Mots-clés : nomenclature, nomenclature, industrie, industrie, catégorie, catégorie, secteur, secteur, branche, branche, Grèce, Grèce, spécificité méditerranéenne, spécificité méditerranéenne, recensement, recensement, théories économiques, théories économiques, désindustrialisation, désindustrialisation, époque contemporaine, époque contemporaine, histoire économique, histoire économique, histoire sociale, histoire sociale

\section{AUTEUR}

VINCENT GOUZI

CREE/Inalco/UPSC 